\title{
Tomato hypersensitivity in peach allergic patients: rPru p3 and rPru p1 positivity is predictive of the symptom severity
}

\author{
Marta Piantanida ${ }^{1 *}$, Larua Farioli ${ }^{2}$, Joseph Scibilia ${ }^{3}$, Ambra Mascheri $^{3}$, Valerio Pravettoni ${ }^{1}$, Laura Primavesi ${ }^{1}$, \\ Michele Nichelatti ${ }^{4}$, Alessandro Marocchi ${ }^{2}$, Jan Walter Schroeder ${ }^{3}$, Chrysi Stafylaraki ${ }^{3}$, Elide Anna Pastorello ${ }^{3}$
}

From Food Allergy and Anaphylaxis Meeting 2011

Venice, Italy. 17-19 February 2011

\section{Background}

allergy to tomato is very common in the Mediterranean area, where tomato consumption has increased in the last years: fresh in salads, cooked in household sauces or industrially processed foods. Many patients allergic to tomato present severe reactions and have symptoms also with commercial tomato products.

\section{Objective}

to examine the possibility of a relationship between severe allergic symptoms to tomatoes and peach; and, in the event of such a relationship, examine the correlation between severe tomato allergy and IgE positivity to peach allergens, i.e. Pru p 3 and Pru p 1.

\section{Methods}

within a population of patients with different grades of OAS for peach (mild-OAS, group A; and severe systemic symptoms to peach, group B) we selected patients with documented allergic reactions to tomato. We investigated the type of reaction to tomato by means of a clinical questionnaire, skin prick tests, prick + prick with fresh tomato and anti-rPru p 1, 3, 4, anti-rBet $\mathrm{v} 1$, 2 and 4 IgE specific measurements. We compared the kind of clinical reactions to tomato between group A and group B patients. Statistical analyses were carried out with parametric and non parametric tests to examine the relationship between anti-rPru p 1 and 3 IgE levels and symptom severity.

\section{Results}

patients with severe symptoms to peach (group B) were at major risk of presenting severe symptoms to tomato $(\mathrm{p}=0.017)$. We investigated this correlation and found that patients with systemic severe symptoms to tomato presented higher specific IgE levels to rPru p 3 than patients with mild OAS $(\mathrm{p}=0.0291)$. On the contrary patients with mild OAS to tomato presented higher rPru p 1 specific IgE levels than patients with severe systemic symptoms $(\mathrm{p}=0.047)$.

\section{Conclusions}

peach and tomato allergy are strictly interrelated and IgE positivity to rPru p 3 can be considered a biological marker of severe symptoms to tomato, whereas IgE to rPru p1 can be considered a marker for milder symptoms.

\section{Author details}

${ }^{1}$ Foundation IRCCS Ca' Granda Ospedale Maggiore Policlinico, Clinical Allergy and Immunology Unit, Milan, Italy. ${ }^{2}$ Niguarda Hospital, Laboratory Medicine, Milan, Italy. ${ }^{3}$ Niguarda Hospital, Allergology and Immunology, Milan, Italy.

${ }^{4}$ Niguarda Hospital, Center for Clinical Research, Milan, Italy.

Published: 12 August 2011

\section{doi:10.1186/2045-7022-1-S1-P77}

Cite this article as: Piantanida et al:: Tomato hypersensitivity in peach allergic patients: rPru p3 and rPru p1 positivity is predictive of the symptom severity. Clinical and Translational Allergy 2011 1(Suppl 1):P77. 